\title{
Influence of the difference of breastfeeding volume on a rat model of oxygen-induced retinopathy
}

\author{
Michiko Matsubara, ${ }^{1}$ Yuta Saito, ${ }^{1, *}$ Takako Nakanishi-Ueda, ${ }^{2}$ Toshihiko Ueda, ${ }^{1}$ Tadashi Hisamitsu, ${ }^{2}$ \\ Ryohei Koide ${ }^{1}$ and Haruo Takahashi ${ }^{1}$ \\ ${ }^{1}$ Department of Ophthalmology and 2Department of Physiology, Showa University School of Medicine, 1-5-8 Hatanodai, Shinagawa-ku, Tokyo 142-8666, Japan
}

(Received 19 April, 2014; Accepted 30 April, 2014; Published online 31 July, 2014)

This study aimed to investigate the effects of abundant breast milk intake on rats model of oxygen-induced retinopathy (OIR). Neonatal Sprague-Dawley rats were randomly assigned to expand litters of 7 pups/litter (7-rats group) and 14 pups/litter (14-rats group). They were exposed to $80 \%$ oxygen from postnatal day (P) 0 to P12. Body weights were measured daily. At P13 and 18, rats were sacrificed, and the blood and eyes were collected. Retinal neovascularization (NV) score, total retinal area (TRA), avascular area (AVA), and vascularized area (VA) were measured in ADPase stained retinas. Retinal vascular endothelial growth factor (VEGF) and serum insulin-like growth factor (IGF-1) were measured using ELISA. Body weight gain was significantly greater in 7-rats group from P2. Serum IGF-1 levels at P13 and 18 were significantly higher in 7-rats group. Retinal VEGF and TRA at P18 were significantly larger in 7-rats group. NV score at P18 tended to be higher in 7-rats group. There was no significant difference in VA between the 2 groups at P13 and 18. Excess breast milk intake in OIR rat pups caused body weight gain and retinal development, whereas there was less effect on retinal vascularization in our study.

Key Words: nutrition, retinopathy of prematurity, oxygen-induced retinopathy, vascular endothelial growth factor, insulin-like growth factor

$\mathrm{R}$ etinopathy of prematurity (ROP) is a leading cause of childhood blindness worldwide. ROP involves two discrete phases. In phase 1 , the retina is relatively hyperoxic with decreased vascular endothelial growth factor (VEGF) levels, whereas in phase 2 , the retina is relatively hypoxic with increased VEGF levels. ${ }^{(1)}$ Currently, ROP is treated with photocoagulation and cryotherapy in phase 2 to decrease the VEGF levels. These treatments require the destruction of the peripheral retina that may cause permanent loss of the peripheral visual field and induction of significant myopia. More recently, the benefits of intravitreal bevacizumab monotherapy for ROP was reported. ${ }^{(1)}$ However, injected bevacizumab can escape from the eye into the systemic circulation and cause systemic complications. ${ }^{(2)}$ Therefore, novel treatments for the prevention of ROP in phase 1 are under evaluation. Over the past decades, the number of infants with ROP has increased because of the increased survival rates of very low birth weight infants. ${ }^{(3)}$ Mean serum insulin-like growth factor-1 (IGF-1) levels in premature infants are directly correlated with the severity of clinical ROP. ${ }^{(4,5)}$ Research has shown that the sufficient intake of nutrients may increase serum IGF-1 levels and may be beneficial in preventing ROP. ${ }^{(4)}$ Human breast milk increases the circulating IGF-1 levels more than supplementation with formula. ${ }^{(6)}$ Nutrition management for very low birth weight infants has dramatically changed since the 2000s; early aggressive nutrition therapy has been shown to prevent extrauterine growth restriction and anomalous development of preterm infants. ${ }^{(7-9)}$ Furthermore, this aggressive nutrition therapy increases serum IGF-1 levels, which may prevent ROP development. ${ }^{(10)}$ However, there are few clinical reports on the effects of aggressive nutrition therapy for preterm infants with ROP.

Consequently, we investigated the effects of abundant breast milk intake on weight gain, retinopathy, retinal VEGF levels, and serum and retinal IGF-1 levels in a rat model of oxygen-induced retinopathy (OIR).

\section{Methods}

Animal model. All experiments were performed in accordance with the ARVO Statement for the Use of Animals in Ophthalmic and Vision Research. All experiments were approved by The Institutional Committee of Animal Care and Use at Showa University Institution (Tokyo, Japan). Female Sprague-Dawley rats at 14 days gestation were purchased from CREA Japan (Tokyo, Japan). Within approximately $6 \mathrm{~h}$ of birth, the neonatal rat pups were randomly divided into 2 groups as follows: each dam was given 7 pups/litter (7-rats group) or 14 pups/litter (14-rats group). It is previously reported that average litter size, which one rat dam gives birth, was from 6 to 14 pups in general. ${ }^{(11,12)}$ Therefore we set 14 pups/litter for maximum average litter size, and $7 \mathrm{pups} /$ litter for half number of maximum average litter size to discriminate breastfeeding volume between each litter. To create OIR model, the two groups were exposed to daily cycles of $80 \%$ oxygen $(20.5 \mathrm{~h})$, ambient air $(0.5 \mathrm{~h})$, and a progressive return to $80 \%$ oxygen $(3 \mathrm{~h})$ in an Oxycycler (Biosperix, NY) from postnatal day (P) 0 to $\mathrm{P} 12$. The rats were then placed in ambient air until P18. The dams were rotated among the groups every two days. ${ }^{(13)}$ Body weight of rat pups was measured every day.

Blood samples and retina processing. At $\mathrm{P} 13$, and 18 , the rat pups were sacrificed by intraperitoneal (IP) injection of sodium pentobarbital $(0.65 \mathrm{mg} / \mathrm{g}$ body weight). Blood samples were collected from the left ventricle and centrifuged at $10,000 \mathrm{rpm}$ for 5 min at $4^{\circ} \mathrm{C}$. The serum samples were stored at $-80^{\circ} \mathrm{C}$ for ELISA testing. Both eyes were enucleated, and the right eyes were fixed for $48 \mathrm{~h}$ in $4 \%$ paraformaldehyde in cacodylate buffer $(0.1 \mathrm{M}$, $\mathrm{pH}$ 7.2). The cornea, lens, and sclera were removed under a microscope and the retina was stained with adenosine diphosphatase (ADPase). The intensity of neovascularization (NV) in each

*To whom correspondence should be addressed. E-mail: s-yuta@showa-u.ac.jp 
A

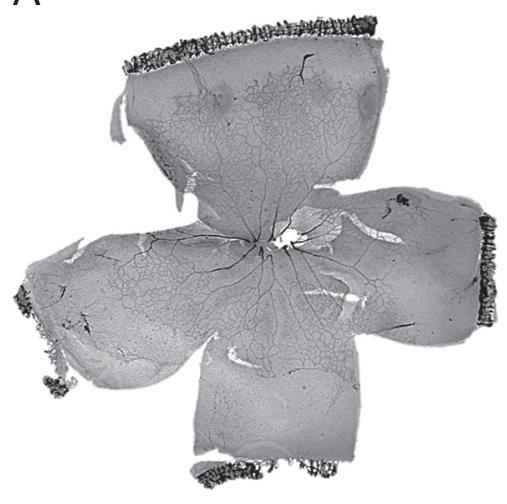

B

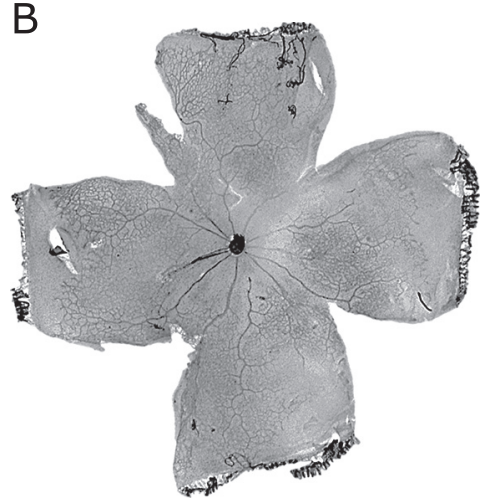

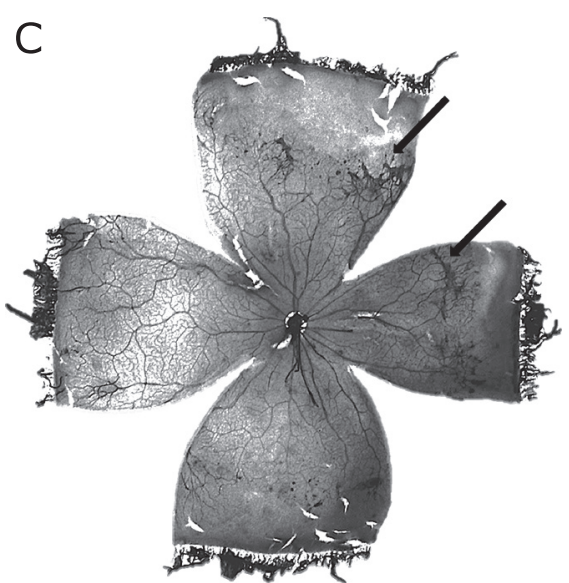

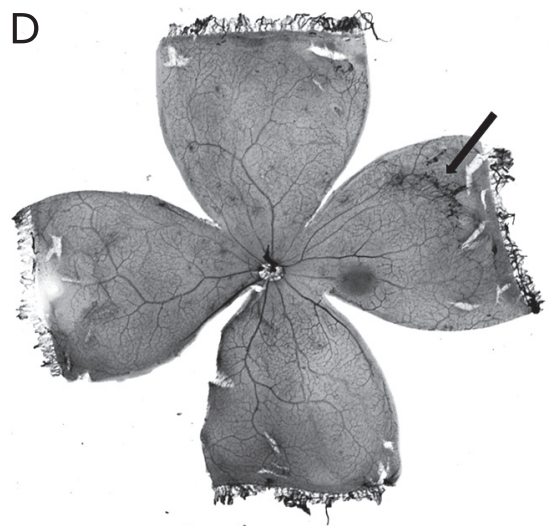

Fig. 1. Digital images of typical stained retinal flat mounts. (A) 7-rats group at P13. \%AVA was 29.0\%. (B) 14-rats group at P13. \%AVA was 15.1\%. (C) 7-rats group at P18. NV score was 7, and \%AVA was $15.5 \%$. (D) 14-rats group at P18. NV score was 4, and \%AVA was $2.2 \%$. Short ridges were found in (C) and (D) (black arrows).

retina was determined as a total of individual quadrant scores using previously described methods (e.g., score 4, long ridge; 3 , short ridge; 2 , five or more glomerular buds; 1, less than five glomerular buds; and 0 , none observed)..$^{(14,15)}$ The retinas were then flat-mounted as previously described. ${ }^{(14,16)}$ Digital images of the flat-mounted retinas were obtained using a D100 (Nikon, Tokyo, Japan). The total retinal areas (TRA) and peripheral avascular area (AVA) were measured using Image $\mathrm{J}$ software (NIH, Bethesda, MD). The vascularized area (VA) in the retina was calculated as the TRA less the peripheral AVA. The peripheral AVA was expressed as a percentage of the TRA (\%AVA). Fig. 1 shows typical digital image of flatmounted ADPase stained retina.

Fresh tissue preparation. The enucleated left eyes were dissected under a microscope. The retinas were isolated and placed in $100 \mu \mathrm{l}$ of tissue protein-extraction reagent (T-PER, Pierce, Chicago, IL) with a protease inhibitor cocktail (1:100, Sigma-Aldrich, St. Louis, MO). The tissue was homogenized and the lysate was centrifuged at $10,000 \mathrm{rpm}$ for $10 \mathrm{~min}$ at $4^{\circ} \mathrm{C}$. The supernatant was collected and stored at $-80^{\circ} \mathrm{C}$ for ELISA testing.

VEGF and IFG-1 measurements. The Quantikine rat VEGF Immunoassay kit (R\&D systems, Minneapolis, MN) was used to measure retinal VEGF levels, while the Quantikine rat IGF-1 Immunoassay kit (R\&D systems) was used to measure serum IGF-1 levels according to the manufacturer's protocol. Total protein was quantified using a BCA protein assay reagent kit (Thermo Fisher Scientific, Waltham, MA) using bovine serum albumin as the standard.
Statistical analysis. The Mann-Whitney $U$ test was used to test for differences between the two groups. Spearman rank correlation coefficients were used to determine the relationships between the mean variables. Data were analyzed with SPSS ver. 20.0 software (Chicago, IL). The significance level was set at $p<0.05$, and the data points were reported as means \pm SE.

\section{Results}

In total, 42 pups were examined (7-rats, $n=14$; 14-rats, $n=28$ ). Fig. 2 shows that there was not significant difference between the body weight of 7-rats and 14-rats groups at P1 $(p=0.495)$. But from $\mathrm{P} 2$, the body weight of 7-rats was significantly bigger than that of 14-rats groups until the end of the study $(p<0.0001)$.

Seven retinal and serum samples from each group at each time point were used for analysis. In 14-rats groups, 7 samples were randomly chosen.

Retinal VEGF levels ( $\mathrm{pg} / \mathrm{mg}$ protein) were relatively higher in 7 -rats $(380.7 \pm 56.5)$ than in 14-rats group $(259.9 \pm 29.6)$ at P13 $(p=0.142)$, and significantly higher in 7-rats $(60.8 \pm 9.2)$ than in 14-rats group $(35.0 \pm 5.5)$ at $\mathrm{P} 18(p<0.05)$ (Fig. $3 \mathrm{~A})$.

Serum IGF-1 levels $(\mathrm{ng} / \mathrm{ml})$ were significantly higher in 7-rats than in 14-rats group at both P13 $(264.3 \pm 21.1$ vs $179.3 \pm 15.8$ : $p<0.05)$ and $\mathrm{P} 18(309.3 \pm 17.5$ vs $171.4 \pm 16.1: p<0.01)$ (Fig. $3 \mathrm{~B}$ ).

Seven flat-mounted retina samples from 7 -rat groups and 14 samples from 14-rat groups obtained at each time point were used for this analysis to measure TRA $\left(\mathrm{mm}^{2}\right), \% A V A, V A\left(\mathrm{~mm}^{2}\right)$, and NV score. TRA $\left(\mathrm{mm}^{2}\right)$ was significantly larger in 7-rats than 


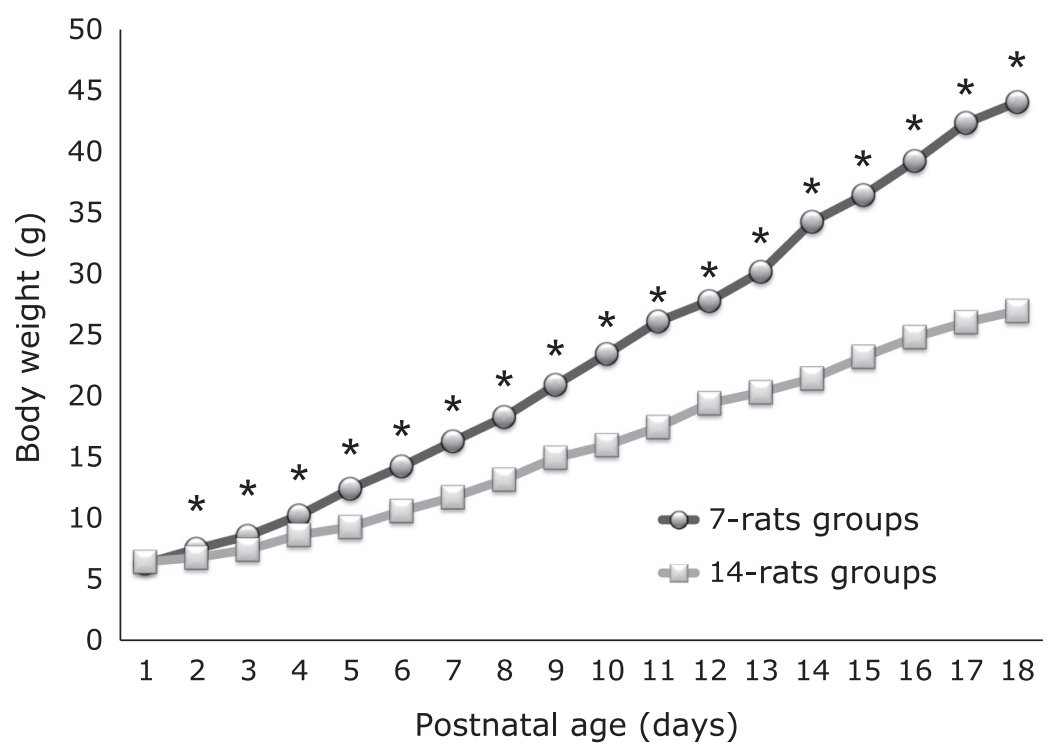

Fig. 2. Postnatal body weight gain. There was no significant difference between 7-rats and 14-rats groups at $P 1$. From $P 2$, there was significant difference between 2 groups ( $\left.{ }^{*} p<0.001\right)$. Data are expressed as mean \pm SEM (At P1: $n=14$ in the 7-rats groups, $n=28$ in the $14-$ rats groups).
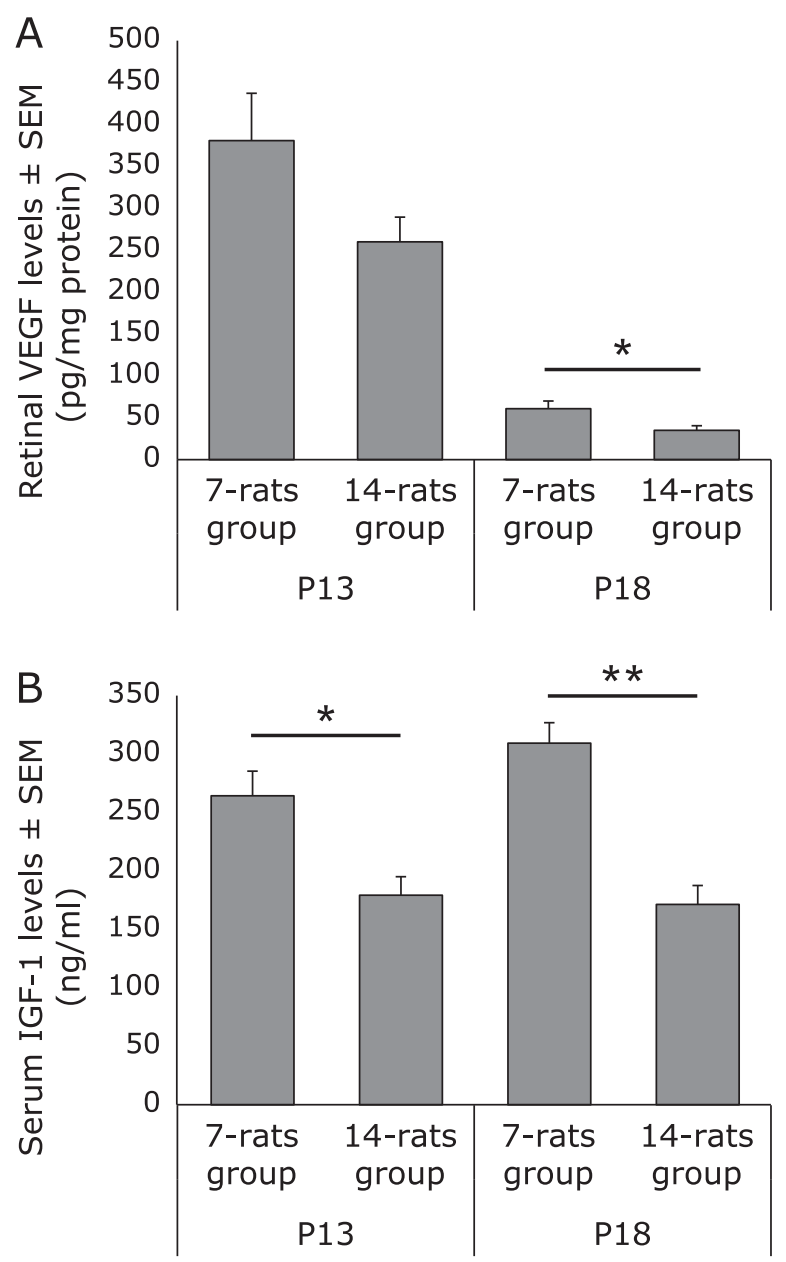

Fig. 3. Transitional change in retinal VEGF (A) and serum IGF-1 (B) levels. Data are expressed as mean \pm SEM $(n=7$ for each of the groups in $(A)$ and $(B)) .{ }^{*} p<0.05, * * p<0.01$. in 14-rats groups at both $\mathrm{P} 13(42.7 \pm 1.5$ vs $38.5 \pm 0.9: p<0.05)$ and P18 (43.7 \pm 0.7 vs $41.5 \pm 0.6: p<0.05)$ (Fig. $4 \mathrm{~A})$.

$\% A V A$ was relatively larger in 7-rats than in 14-rats groups at P13 (40.3 \pm 5.3 vs 27.6 $\pm 2.2: p=0.062)$ and P18 (10.4 \pm 3.2 vs $4.1 \pm 1.1: p=0.113)$, but no statistical significance was detected between two groups (Fig. 4B). There was not significant difference in VA $\left(\mathrm{mm}^{2}\right)$ between 7-rats and 14-rats groups at either P13 $(25.4 \pm 2.4$ vs $27.9 \pm 1.2)$ or P18 $(39.3 \pm 2.0$ vs $39.8 \pm 0.8)$ (Fig. 4C).

Retinal NV only appeared at P18. The average NV score in the 7 -rats group ( $4.9 \pm 1.4)$ was higher than that in the 14-rats group $(2.6 \pm 0.7)$. But there was no statistical significance (Fig. 4D).

\section{Discussion}

Nutritional intake of rat pups is entirely dependent on breast milk from their dams until P20; ${ }^{(17)}$ therefore, their body weight gain should be logically impacted by the amount of milk intake. In humans, ROP risk has been associated with low circulating IGF-1 levels and slow postnatal weight gain. ${ }^{(14,18,19,20)}$ In mice experiments, it is reported that postnatal body weight gain may prevent the deterioration of retinopathy. ${ }^{(21,22)}$ IGF-1 is related to nutrition supply, which is essential for both pre- and postnatal general growth as well as growth of the retinal vasculature. ${ }^{(23,24)}$

We conducted this research to investigate the effects of abundant breast milk intake on weight gain, retinopathy, retinal VEGF levels, and serum IGF-1 levels in of OIR rat model. We had previously used $12-14$ rat pups/litter as a standard OIR litter. ${ }^{(13,25-28)}$ On the basis of these reports and average litter size that were reported by Taniguchi ${ }^{(11)}$ and Imamichi, ${ }^{(12)}$ we redistributed the pups litters at P0 among dams, i.e., smaller litters (7-rats groups) and larger litters (14-rats groups). Seven-rats groups were expected to be overfeeding. The rat OIR model is widely used as a mimic of clinical ROP. ${ }^{(14,16,29,30)}$ This model comprises 2 phases. First, there is a suppression of retinal vessel development because of a decrease in growth factors, including VEGF, during hyperoxia. Second, there is an increase in VEGF levels during the development of peripheral avascular areas in the retina.

Dhaliwal et al. ${ }^{(29)}$ reported that the retinal vessels in normal rat pups nearly vascularized at P14. But retinal vessels in rats do not normally develop when they are exposed in high concentration of 

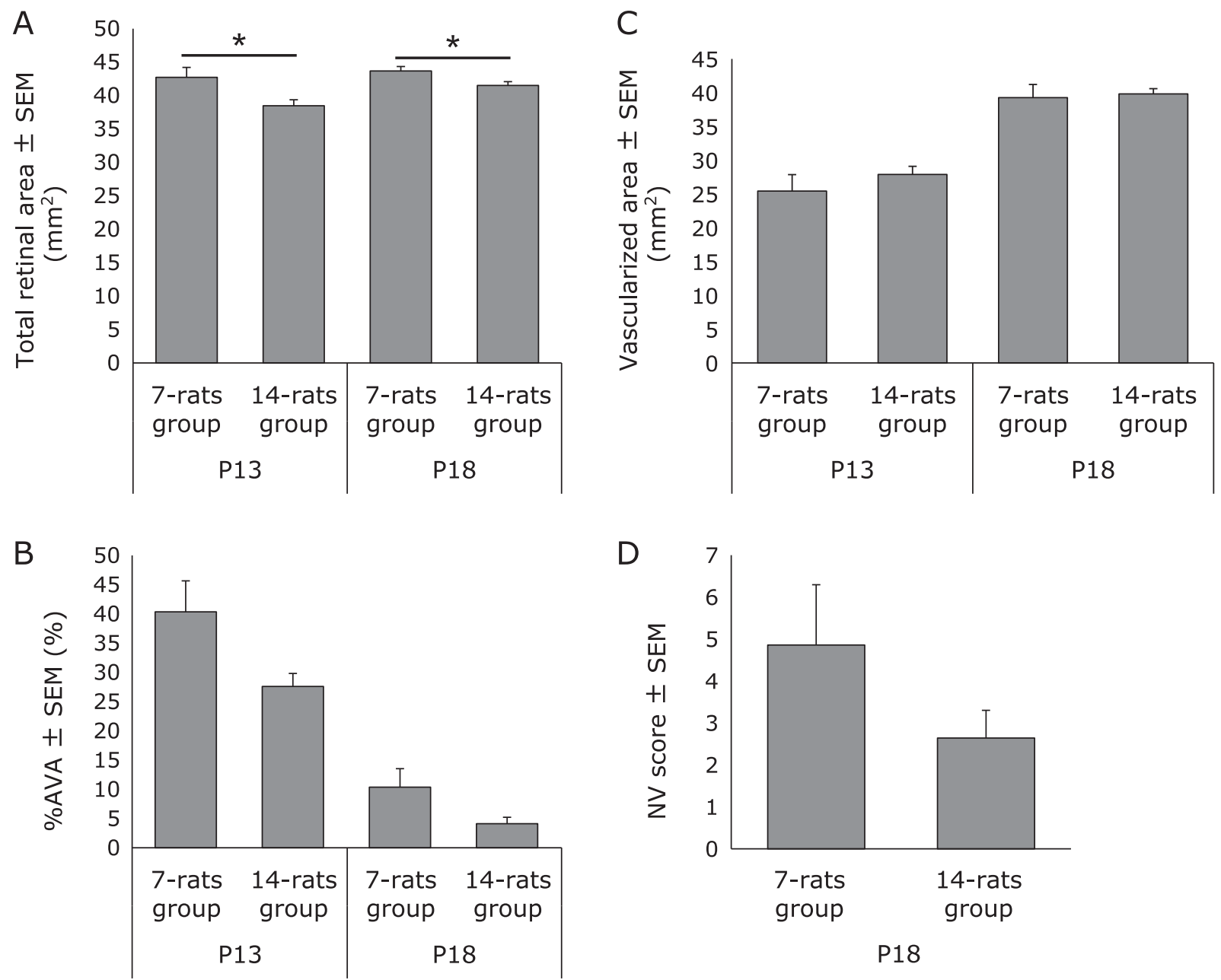

Fig. 4. Transitional change in TRA (A), \%AVA (B), VA (C) and NV scores (D). Data are expressed as mean \pm SEM $(n=7$ in the 7-rat groups and $n=14$ in the 14-rat groups at each time point). ${ }^{*} p<0.05$. \%AVA in 7-rats group was relatively larger than that of 14-rats group at both P13 and P18, but there was no significant difference (B). There was no significant difference in (C) or in (D).

oxygen. In our study, retinal VEGF levels in OIR are expected to increase significantly at $\mathrm{P} 13$ when it is $24 \mathrm{~h}$ after the completion of oxygen exposure, and extraretinal neovascularization was observed at P18. Hellstrom et al. ${ }^{(22)}$ reported that serum IGF-1 levels were related to nutrition supply. In our study, serum IGF-1 levels significantly correlated with body weight at P13 and P18 $(r=0.7155, p<0.0001$, Fig. 5). From them, it would be inferred that body weight and serum IGF-1 levels between 7-rats and 14rats groups were influenced by the difference of breastfeeding volume. In addition, the correlation coefficient between body weight and TRA was significant $(r=0.5032, p<0.0001$, Fig. 6), suggesting rat's eyeball itself have developed with the body weight gain. Whereas, VA did not have significant difference between 2 groups, which suggests that the speed of retinal vascular development may not significantly differed in both groups. Taken together, it is considered that 7-rats groups had relatively larger AVA than 14-rats groups, which caused higher retinal VEGF levels in 7-rats groups at P18. Holmes et al. ${ }^{(31,32)}$ reported that the litter size affected normal retinal vascular development in normal neonatal rats and abnormal neovascularization in OIR rats. They resulted that VA were significantly greater in the smaller litters (10 pups/litter) than in the larger litters (18 pups/ litter). On the other hand, we set 7 pups/litter as a smaller litter and $14 \mathrm{pups} /$ litter as a larger litter, which the number of pups was within limits of an average litter size. Eighteen pups/litter were possibly so undernourished that lead to the vascular under-

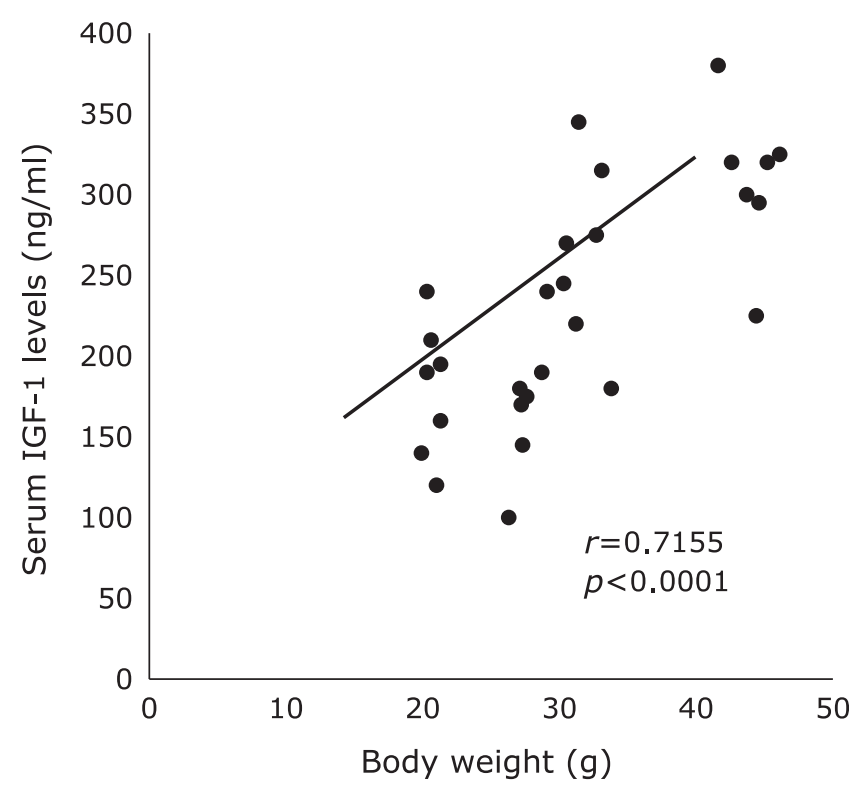

Fig. 5. A positive significant correlation was noted between body weight and serum IGF-1 levels $(r=0.7155, p<0.0001)$. Scatter plot included the data at P13 and P18 in 7-rat and 14-rat groups. 


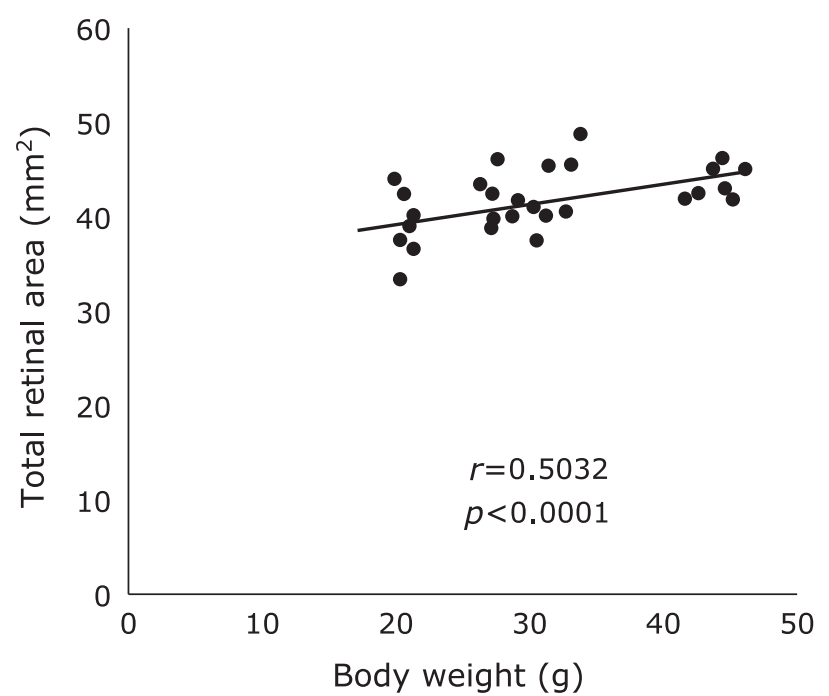

Fig. 6. A positive significant correlation was noted between body weight and TRA $(r=0.5032, p<0.0001)$. Scatter plot included the data at P13 and P18 in 7-rat and 14-rat groups.

development. Therefore, their findings were different from our result. Otherwise, Stahl et al. ${ }^{(20)}$ reported that OIR was more severe in mice with poor weight gain than moderate or extensive weight gain. These differences may be based on the period of oxygen exposure, litter size, or the use of different animal models such as mice and rats. They used larger litters as groups that received lesser nutrition, whereas we used them as normal nutrition groups. Because our purpose was to determine whether early aggressive nutrition had an effect on OIR, we used smaller litters as the higher nutrition groups. Although this has not clarified the relationship between neonatal nutrition intake and ocular development, considering the findings in our OIR rat model, there is a possibility that abundant nutrition may increase the severity of OIR.

In our study, NV score at P18 in 7-rats was relatively higher than that of 14-rats group. NV score of each animal varied widely, and number of samples might not be enough in our study. That may be the reason why there was not significant difference in the two groups. The limitation of this study is that this rat model is somewhat different from OIR in premature infants. Low gestational age and being small for gestational age (SGA) increase the risk for severe ROP. ${ }^{(23)}$ In contrast, the rat pups in this study were born as term newborns and were of appropriate size for their gestational age. An OIR rat model using SGA rat pups may demonstrate a different phenomenon. ${ }^{(29)}$ In addition, it is important to consider the number of pups in a litter when an OIR model is used because different numbers of pups in a litter can affect the severity of OIR.

In conclusion, excess breast milk intake in OIR rat pups caused body weight gain and retinal development, whereas there was less effect on retinal vascularization in our study.

\section{Acknowledgments}

We thank Ms. Ozawa for her technical assistance. This work was supported by JSPS KAKENHI 25870737.

\section{Conflict of Interest}

No potential conflicts of interest were disclosed.

\section{References}

1 Mintz-Hittner HA, Kennedy KA, Chuang AZ. Efficacy of intravitreal bevacizumab for stage 3+ retinopathy of prematurity. N Engl J Med 2011; 364: 603615.

2 Sato T, Wada K, Arahori H, et al. Serum concentrations of bevacizumab (avastin) and vascular endothelial growth factor in infants with retinopathy of prematurity. Am J Opthalmol 2012; 153: 327-330.

3 Chen J, Smith LE. Retinopathy of prematurity. Angiogenesis 2007; 10: $133-$ 140.

4 Hellström A, Engström E, Hård AL, et al. Postnatal serum insulin-like growth factor I deficiency is associated with retinopathy of prematurity and other complications of premature birth. Pediatrics 2003; 112: 1016-1020.

5 Hellstrom A, Perruzzi C, Ju M, et al. Low IGF-I suppresses VEGF-survival signaling in retinal endothelial cells: direct correlation with clinical retinopathy of prematurity. Proc Natl Acad Sci U S A 2001; 98: 5804-5808.

6 Díaz-Gómez NM, Domenech E, Barroso F. Breast-feeding and growth factors in preterm newborn infants. J Pediatr Gastroenterol Nutr 1997; 24: $322-327$.

7 Thureen PJ. Early aggressive nutrition in very preterm infants. Nestle Nutr Workshop Ser Paediatr Program 2007; 59: 193-204; discussion 204-208.

8 Ehrenkranz RA. Early, aggressive nutritional management for very low birth weight infants: what is the evidence? Semin Perinatol 2007; 31: 48-55.

9 Hay WW Jr. Strategies for feeding the preterm infant. Neonatology 2008; 94 : 245-254.

10 Can E, Bülbül A, Uslu S, Cömert S, Bolat F, Nuhoğlu A. Effects of aggressive parenteral nutrition on growth and clinical outcome in preterm infants. Pediatr Int 2012; 54: 869-874.

11 Taniguchi H. Laboratory Animal Science. 8. Tokyo: Ishiyaku Publisher, Inc., 1997; 8-9.

12 Imamichi T. Series of Experimental Animals. Vol. 2. Rearing Management and Manipulation of Experimental Animals. Tokyo: Soft Science, Inc., 1979; 247-251.

13 Dorey CK, Aouididi S, Reynaud X, Dvorak HF, Brown LF. Correlation of vascular permeability factor/vascular endothelial growth factor with extra- retinal neovascularization in the rat. Arch Ophthalmol 1996; 114: 1210-1217.

14 Hasebe Y, Thomson LR, Dorey CK. Pentoxifylline inhibition of vasculogenesis in the neonatal rat retina. Invest Ophthalmol Vis Sci 2000; 41: 27742778.

15 Reynaud X, Dorey CK. Extraretinal neovascularization induced by hypoxic episodes in the neonatal rat. Invest Ophthalmol Vis Sci 1994; 35: 3169-3177.

16 Modanlou HD, Gharraee Z, Hasan J, Waltzman J, Nageotte S, Beharry KD. Ontogeny of VEGF, IGF-I, and GH in neonatal rat serum, vitreous fluid, and retina from birth to weaning. Invest Ophthalmol Vis Sci 2006; 47: 738744.

17 Löfqvist C, Andersson E, Sigurdsson J, et al. Longitudinal postnatal weight and insulin-like growth factor I measurements in the prediction of retinopathy of prematurity. Arch Ophthalmol 2006; 124: 1711-1718.

18 Wallace DK, Kylstra JA, Phillips SJ, Hall JG. Poor postnatal weight gain: a risk factor for severe retinopathy of prematurity. JAAPOS 2000; 4: 343-347.

19 Allegaert K, Vanhole C, Casteels I, et al. Perinatal growth characteristics and associated risk of developing threshold retinopathy of prematurity. JAAPOS 2003; 7: 34-37.

20 Stahl A, Chen J, Sapieha P, et al. Postnatal weight gain modifies severity and functional outcome of oxygen-induced proliferative retinopathy. Am J Pathol 2010; 177: 2715-2723.

21 Vanhaesebrouck S, Daniëls H, Moons L, Vanhole C, Carmeliet P, De Zegher F. Oxygen-induced retinopathy in mice: amplification by neonatal IGF-I deficit and attenuation by IGF-I administration. Pediatr Res 2009; 65: 307310 .

22 Hellström A, Ley D, Hansen-Pupp I, et al. New insights into the development of retinopathy of prematurity-importance of early weight gain. Acta Paediatr 2010; 99: 502-508.

23 Hansen-Pupp I, Löfqvist C, Polberger S, et al. Influence of insulin-like growth factor I and nutrition during phases of postnatal growth in very preterm infants. Pediatr Res 2011; 69: 448-453.

24 Kabayama J, Saito Y, Nakanishi-Ueda T, et al. Effects of subconjunctival injection of anti-vascular endothelial growth factor antibody on oxygen- 
induced ischemic retinopathy in a neonatal rat model. The Showa Univ J Med Sci 2012; 24: 11-19.

25 Tomoyasu-Okamoto S, Nakanishi-Ueda T, Saito Y, et al. Effect of Ethylene Diurea on Oxygen-induced Ischemic Retinopathy in the Neonatal Rat. The Showa Univ J Med Sci 2010; 22: 41-50.

26 Saito Y, Hasebe-Takenaka Y, Ueda T, et al. Effects of green tea fractions on oxygen-induced retinal neovascularization in the neonatal rat. J Clin Biochem Nutr 2007; 41: 43-49.

27 Saito Y, Uppal A, Byfield G, Budd S, Hartnett ME. Activated NAD(P)H oxidase from supplemental oxygen induces neovascularization independent of VEGF in retinopathy of prematurity model. Invest Ophthalmol Vis Sci 2008; 49: 1591-1598.

28 Leske DA, Wu J, Fautsch MP, et al. The role of VEGF and IGF-1 in a hyper- carbic oxygen-induced retinopathy rat model of ROP. Mol Vis 2004; 10: 4350 .

29 Dhaliwal CA, Wade J, Gillespie T, Aspinall P, McIntosh N, Fleck BW. Early retinal blood vessel growth in normal and growth restricted rat pups raised in oxygen and room air. Br J Ophthalmol 2011; 95: 1592-1596.

30 Raghuveer TS, Bloom BT. A paradigm shift in the prevention of retinopathy of prematurity. Neonatology 2011; 100: 116-129.

31 Holmes JM, Duffner LA. The effect of litter size on normal retinal vascular development in the neonatal rat. Curr Eye Res 1995; 14: 737-740.

32 Holmes JM, Duffner LA. The effect of postnatal growth retardation on abnormal neovascularization in the oxygen exposed neonatal rat. Curr Eye Res 1996; 15: 403-409. 